\title{
Die Defizitkriterien des Europäischen Primärrechts
}

von Lars Dittrich

\section{Grundgedanken der Normierung}

Die Einführung einer gemeinsamen Währung ist integraler Teil der europäischen Einigung $^{1}$ und als solcher im Zielkatalog des EUV normiert (Art. 3 Abs. 4 EUV). ${ }^{2}$ Historisch war die deutsche Vorbedingung für eine gemeinsame europäische Währung die Absicherung ihrer Stabilität. Als Garanten dieser Stabilität sah man auf die Geldwertstabilität verpflichtete, unabhängige Zentralbanken (Art. 127 Abs. 1, Art. 282 Abs. 2 S. 2 sowie vor allem Art. 130, 131 AEUV) und "gesunde" Staatsfinanzen an. ${ }^{3}$

Den Art.119 ff. AEUV liegt der Gedanke zu Grunde, dass Mitgliedstaaten mit expansiver Staatsverschuldung einen immer größeren Teil ihrer Einnahmen zur Tilgung der Zinsen aufbringen müssen. So bleiben ihnen immer weniger Mittel zur Gestaltung ihrer eigentlichen politischen Ziele. Um ihren Handlungsspielraum wieder zu erhöhen, bieten sich ihnen drei Möglichkeiten. Entweder sie nehmen neue Schulden auf. Das führt letztlich in einen circulus vitiosus, zumal mit steigender Verschuldung die Wahrscheinlichkeit steigt, dass der Schuldnerstaat seine Verbindlichkeiten nicht mehr bedienen kann. Dieses Risiko kalkulierend, verlangen die Gläubiger höhere Risikoaufschläge für ihre Anleihen, wodurch wiederum die Refinanzierungskosten des Mitgliedstaates steigen. Am Ende dieser Entwicklung steht der Staatsbankrott oder das Bedürfnis nach finanzieller Hilfe anderer Staaten oder Internationaler Organisationen.

Die zweite Möglichkeit, politische Handlungsfähigkeit zurückzugewinnen, besteht für die Mitgliedstaaten darin, ihre Ausgaben zu kürzen. Ausgabenkürzungen treffen jedoch das Wahlvolk und sind deshalb bei diesem und den von ihnen

1 „L'Europe se fera par la monnaie ou ne se fera pas.“ - Jacques Rueff, zitiert nach Selmayr, M.: Das Recht der Wirtschafts- und Währungsunion, München, 2002, V.

2 Khan, D.E.: Art.126 AEUV, Rn.1., in: Geiger/Khan/Kotzur: EUV/AEUV Kommentar, München, 2010.

3 Ausführliche Darstellung aus Sicht der Zentralbank bei Issing, O.: Der Euro, München, 2008, 49. 
Gewählten und Wiederzuwählenden unpopulär. Auf der Suche nach Alternativen bleibt ihnen ein für die Wähler ,unsichtbarer“" ${ }^{\star 4}$ bzw. weniger spürbarer dritter Ausweg: eine inflationäre Geldpolitik. In deren Folge bleibt zwar der Nominalwert der Währung und damit auch der Schulden in dieser Währung gleich, ihr Realwert sinkt jedoch. ${ }^{5}$ Damit sinkt auch der Wert der in dieser Währung gemessenen Staatsschulden und der Staat gewinnt politische Handlungsfähigkeit zurück.

\section{Historische Entwicklung}

Um inflationsgeneigte Geldpolitik zu vermeiden, mussten alle Teilnehmer an der Währungsunion die sog. Maastricht-Kriterien (Inflation max. 1,5\% über jener der drei inflationsstabilsten Mitgliedstaaten, max. 3\% öffentliches Defizit, max. 60\% Gesamtschuldenstand) erfüllen. ${ }^{6}$ Beitrittsvoraussetzung waren damit letztlich eine in bestimmten Grenzen bestehende Stabilitätskultur sowie gesunde Staatsfinanzen.

Um die Stabilität der neuen Währung zu gewährleisten, bedurfte es aber auch nach ihrer Einführung einer soliden Haushaltspolitik. Kompetenzrechtlich ergab sich das Problem, dass die Währungsverantwortung fortan in Europa lag, die Wirtschafts- und Haushaltspolitik aber als nationale Politikbereiche erhalten bleiben sollten. So wurden mit dem Vertrag von Maastricht die „Vermeidung übermäßiger öffentlicher Defizite“ mit den Referenzkriterien ,öffentliches Defizit“" und „Gesamtschuldenstand“ eingeführt, um eine gewisse Haushaltsdisziplin zu gewährleisten und den Mitgliedstaaten trotzdem die Haushaltsautonomie zu erhalten. $^{7}$ Heute ist dies in Art. 126 AEUV (ehemals Art. 104 EGV; davor Art. 104c) i.V.m. Art. 1 Protokoll Nr. 12 „Über das Verfahren bei einem über-

4 Seidel, M.: Währungspolitik als Sozialpolitik, in: Europa und seine Verfassung, Festschrift für Manfred Zuleeg, Baden-Baden, 2005, 505-537, 512.

5 Ob die Gläubiger sich hiergegen mittels sogenannter Wertsicherungsklauseln wehren können, ist im deutschen Recht umstritten. Diese Klauseln binden die Höhe der Rückzahlungsverpflichtung nicht an einen nominalen Geldbetrag, sondern an einen Preisindex. So wird sichergestellt, dass der Gläubiger auch den Realwert seiner Darlehensforderung zurückerstattet bekommt und nicht nur eine nominal identische Summe. Die Zulässigkeit derartiger Klauseln hängt von der strittigen Frage ab, ob sie unter das Verbot des $\S 1$ PrKG fallen und ob diese Regelung unionsrechtlich zulässig ist. Für die Wirksamkeit: Herrmann, C.: Währungshoheit, Währungsverfassung und subjektive Rechte, 359f.; dagegen: Ohler, C.: Die hoheitlichen Grundlagen der Geldordnung, in: Juristenzeitung, 2008, 313-322, 321.

6 Schon bei Einführung der gemeinsamen Währung erfüllten nicht alle Mitgliedstaaten die Kriterien.

7 Bandilla, R.: Art.104 EGV, Rn.1, in: Grabitz/Hilf: Das Recht der Europäischen Union - Kommentar, München, 2007. 
mäßigen Defizit“ geregelt. Gemäß Art. 51 EUV ist auch dieses Protokoll Bestandteil des Primärrechts. Es wird präzisiert durch die VO 3605/93 „Über die Anwendung des Protokolls über das Verfahren bei einem übermäßigen Defizit“.

Mit dem Näherrücken der Euro-Einführung wuchs die Sorge um die Stabilität der gemeinsamen Währung. Deshalb wurden die Vorgaben 1997 auf Bestreben des deutschen Finanzministers Theo Waigel sekundärrechtlich durch zwei Verordnungen VO 1466/97 (Ausbau der haushaltspolitischen Überwachung) und VO 1467/97 (Beschleunigung und Klärung des Verfahrens bei übermäßigem Defizit) und die nur politisch verbindliche Entschließung 97/C 236/01 des Rates präzisiert und verschärft. ${ }^{8}$ Sie bilden zusammen den sogenannten „Stabilitätsund Wachstumspakt". Dieser sollte über Aufsichts- und Sanktionsmaßnahmen die Einhaltung der Defizitkriterien sicherstellen.

Im Jahr 2003 leitete die Kommission Defizitverfahren gegen Deutschland und Frankreich ein. Auf ihre Empfehlung hin stellte der Rat das Bestehen eines übermäßigen Defizits in Deutschland ${ }^{9}$ und Frankreich ${ }^{10}$ fest. Als sich die finanzielle Situation in beiden Ländern nicht besserte, wollte die Kommission den nächsten Schritt des Verfahrens gehen. Sie empfahl dem Rat die bisher in den Staaten getroffenen Maßnahmen als unzureichend zu qualifizieren und beide Staaten mit einer Frist zur Umsetzung einzelner Maßnahmen zur Haushaltskonsolidierung in Verzug zu setzen. ${ }^{11}$ Dafür hätte es einer Abmahnung nach Art. 126 Abs. 9 AEUV bedurft. Sie bindet den Mitgliedstaat und stellt den letzten Schritt vor Einleitung des Sanktionsverfahrens dar. ${ }^{12}$ Der Rat verweigerte diese Abmahnung. Der Versuch der Kommission, diese Entscheidung vor dem EuGH für nichtig erklären zu lassen, scheiterte, weil die reine Nichtannahme von Vorschlägen der Kommission nicht per Nichtigkeitsklage angreifbar ist. ${ }^{13}$

Als Reaktion auf diese Kontroverse entschieden sich die Mitgliedstaaten 2005 für mehr „haushaltspolitische Bewegungsfreiheit“. Dazu änderten Sie die Ver-

8 EuGH, Urteil vom 13.7.2004 - C 27/04 Rn.71; umfassend bei Hentschelmann, K.: Der Stabilitäts- und Wachstumspakt, Baden-Baden, 2009, $205 \mathrm{ff}$.

9 AB1. 2003 L 34/16.

10 AB1. 2003 L165/29.

11 Bandilla, R.: Ist der Stabilitäts- und Wachstumspakt rechtlich durchsetzbar? - Anmerkungen zum Urteil des Gerichtshofes in der Rechtssache Kommission/Rat (C-27-04), in: Europa und seine Verfassung, Festschrift für Manfred Zuleeg, Baden-Baden, 2005, 538-549, 542.

12 Bandilla, R.: Stabilitäts- und Wachstumspakt, a.a.O., 542.

13 EuGH, Urteil vom 13.7.2004 - C 27/04 Leitsatz 1. 
ordnungen des Stabilitäts- und Wachstumspaktes durch VO 1565/05 (Änderung VO 1466/97) und VO 1566/05 (Änderung VO 1467/97).

Der Vertrag von Lissabon stärkte die Rolle der Kommission im Defizitverfahren nur in geringem Ausmaß. Sie unterbreitet dem Rat nun den Vorschlag zur Feststellung des Bestehens eines Defizites, statt der bisherigen „Empfehlung“. Will der Rat diesen ablehnen, ändert sich nichts an der bisherigen Rechtslage, will er ihn aber inhaltlich modifiziert verabschieden, ist das nur einstimmig möglich.

\section{Die Kriterien}

Ein übermäßiges öffentliches Defizit im Sinne von Art. 126 Abs. 1 AEUV liegt vor, wenn die nachhaltige Finanzierbarkeit des öffentlichen Haushalts gefährdet ist oder eine stabilitätswidrig expansive Haushaltspolitik betrieben wird. ${ }^{14} \mathrm{Ob}$ das der Fall ist, lässt sich nur aufgrund einer umfassenden Würdigung der wirtschafts- und haushaltspolitischen Gesamtlage beurteilen. ${ }^{15}$ Die primären Parameter dieser Beurteilung benennt Art. 126 Abs. 2 AEUV mit dem öffentlichen Schuldenstand und dem Finanzierungsdefizit. Art. 1 des Protokolls Nr. 12 legt die bereits dokumentierten Referenzwerte für die Neuverschuldung auf max. 3\% BIP und die Gesamtschuldenstand auf max. 60\% BIP fest.

Art. 126 Abs. 2, 3 AEUV benennen zugleich aber auch Gründe, aus denen das Überschreiten der Stabilitätskriterien gerechtfertigt ist. Nach der Gesamtschau der Normen liegt im Überschreiten der Referenzkriterien daher nicht automatisch ein Vertragsverstoß. ${ }^{16}$ Gleichwohl indizieren sie das Vorliegen eines übermäßigen öffentlichen Defizits.

\section{Notwendigkeit und ökonomische Sinnhaftigkeit fester Referenzkriterien}

Die Wirtschaftstheorie streitet seit jeher über die Notwendigkeit und Grenzen staatlicher Verschuldung. ${ }^{17}$ Im europäischen Primärrecht gilt deren Regelung aber nicht als Selbstzweck, sondern als Voraussetzung einer stabilen Währung. ${ }^{18}$

14 Hattenberger, D.: Art.104 EGV, Rn. 17, in: Schwarze: EU-Kommentar, Baden-Baden, 2009.

15 Khan, D. E., a.a.O., Art.126 AEUV, Rn.7.

16 Hattenberger, D.: a.a.O., Art.104 EGV, Rn.13.

17 Auf der einen Seite sieht bereits Ricardo darin ,eine der schrecklichsten Geißeln, die je zur Plage der Nation erfunden wurden“ - zitiert nach Piel/Simmert: Staatsverschuldung - Schicksalsfrage der Nation?, 1981, 7; gegensätzlicher Ansichten finden sich bei von Stein: „Ein Staat ohne Staatsverschuldung tut entweder zu wenig für seine Zukunft oder er fordert zu viel von seiner Gegenwart“, in: Lehrbuch der Finanzwissenschaft, Leipzig, 1886, 347. 
Die Aussage, dass hohe Staatsschulden zwangsweise zu inflationsfördernder Geldpolitik führen, ${ }^{19}$ setzt logisch voraus, dass die Regierungen auch Einfluss auf die Geldpolitik nehmen können. Gerade das soll die Unabhängigkeit der Europäischen Zentralbank (EZB) und der nationalen Zentralbanken des Europäischen Systems der Zentralbanken (ESZB), Art. 130 S. 1, 2 AEUV, ${ }^{20}$ aber verhindern. $^{21}$

Nach gesicherter ökonomischer und in Folge der Griechenlandkrise auch empirischer Erkenntnis, erhöht ein übermäßiges öffentliches Defizit jedoch den politischen Handlungsdruck auch auf die Notenbanken. Das erschüttert die Glaubwürdigkeit einer auf monetäre Stabilität ausgerichteten Geldpolitik der EZB, belastet die Unabhängigkeitsgarantien ${ }^{22}$ und erschwert so die Gewährleistung der benannten Zielsetzungen. ${ }^{23}$

\section{Sinnhaftigkeit der konkreten Referenzkriterien}

\section{a.) Sinnhaftigkeit von Defizitkriterien}

Die konkreten Werte der Referenzkriterien sind aus wirtschaftswissenschaftlicher Sicht nicht zwingend. ${ }^{24}$ Sie normieren eine als „tragbar“ angesehene Situation. Der rechtliche Maßstab ist also nicht das Wünschenswerte, sondern das noch Finanzierbare. Von ökonomischer Seite ist zudem wiederholt die fehlende Einbeziehung der gegenwärtigen Dynamik und zukünftiger Entwicklungen, etwa der Demographie oder bereits übernommener Pensionsverpflichtungen bemän-

18 Khan, D. E., a.a.O., Art.126 AEUV, Rn.1.

19 Ökonomisch ist dieser Zusammenhang teilweise als ,nicht zwingend“ bezeichnet worden - Ausführliche Darstellung bei Schulze Steinen, M.: Rechtsfragen zur Wirtschaftsunion, Baden-Baden, 1998, 75ff.

20 Vgl. die Darstellung zur Unabhängigkeit u.a. bei Dauses, M.: Rechtliche Grundlagen der Europäischen Wirtschafts- und Währungsunion, München, 2003, 209ff.

21 Pipkorn, J., in: Europarecht 1994, Beiheft 1, 87; weitere ökonomische Begründungsansätze liefert Jarchow, H.-J.: Theorie und Politik des Geldes, Göttingen, 2003, $327 \mathrm{ff}$.

22 Kerber/Städter: Die EZB in der Krise: Unabhängigkeit und Rechtsbindung als Spannungsverhältnis, in: Europäische Zeitschrift für Wirtschaftsrecht, 2011, 536-541, 537; Seidel: Der Ankauf nicht markt- und börsengängiger Staatsanleihen, namentlich Griechenlands, durch die Europäische Zentralbank und durch nationale Zentralbanken - rechtlich nur fragwürdig oder Rechtsverstoß?, in: Europäische Zeitschrift für Wirtschaftsrecht, 2010, 521. Beide sehen in den engen zeitlichen und räumlichen Überschneidungen politischer und zentralbanklicher Aktivitäten in der aktuellen Krise bereits einen Verstoß.

23 Schulze Steinen, M., a.a.O., 221.

24 Schulze-Steinen, M., a.a.O., 203. 
gelt worden. ${ }^{25}$ Wie für die Schuldengrenzen des Grundgesetzes gilt aber auch für den AEUV, dass die Kritik an den bestehenden Regeln nicht von Ihrer Einhaltung und Durchsetzung dispensiert.

\section{b.) Sinnhaftigkeit der ursprünglichen Defizitkriterien}

Die beiden Referenzwerte Neuverschuldung und Gesamtschuldenstand sind interdependent. Mit der Neuverschuldung steigt immer auch der Gesamtschuldenstand. Entscheidend ist jedoch nicht der absolute Gesamtschuldenstand, sondern dessen relativer Wert im Verhältnis zum BIP. Folglich bleibt im Falle von Neuverschuldung der Gesamtschuldenstand nur dann stabil, wenn auch das BIP wächst. Als Ausgangswert für den Gesamtschuldenstand wählten die Mitgliedstaaten den Durchschnitt der nationalen Schuldenstände in der Europäischen Gemeinschaft bei Vertragsfassung, 60\% des BIP. ${ }^{26}$ Bei einer erlaubten Neuverschuldung von 3\% bleibt der Gesamtschuldenstand aber nur dann stabil bei $60 \%$ des BIP, wenn letzteres nominal um 5\% wächst. Bei geringerem Wachstum ergibt sich ein deutlich höherer Gesamtschuldenstand. ${ }^{27}$ Die Bundesrepublik Deutschland als größte Volkswirtschaft der Eurozone erzielte eine derartige Wachstumsrate zuletzt 1994.

Des Weiteren sind Schatten- und Nebenhaushalte nicht von den Defizitkriterien erfasst. ${ }^{28}$ Außerdem sind die nationalen Defizitquoten nur begrenzt vergleichbar, weil nach der Legaldefinition auch Verbindlichkeiten der Sozialversicherungssysteme berücksichtigt werden. ${ }^{29}$ Diese sind aber europaweit unterschiedlich ausgestaltet und wirken, je nachdem ob die Systeme auch zukünftige Ansprüche entstehen lassen, unterschiedlich stark auf die Schuldenquote ein. ${ }^{30}$

Andererseits zeigt das Beispiel Japans mit einer Verschuldensquote von $>200 \%$ BIP, dass je nach Gläubiger- und Schuldenstruktur auch deutlich über

25 Vgl. etwa Raffelhüschen/Moog/Müller: Ehrbare Staaten? Eine deutsche Generationenbilanz im internationalen Vergleich: Wie gut ist Deutschland auf die demographische Entwicklung vorbereitet?, Stiftung Marktwirtschaft, Argumente zu Marktwirtschaft und Politik, 110, Mai 2010.

26 Schulze Steinen, M., a.a.O., 214.

27 Vgl. Berechnungen des Kornberger Kreises: Den Stabilitäts- und Wachstumspakt härten, Frankfurt, $2005,17$.

28 Emmerich-Fritsche, A.: Wie verbindlich sind die Konvergenzkriterien?, Europäisches Wirtschafts- und Steuerrecht, 3 (1996), 77-86, 80.

29 Gem. Art. 2 Protokoll Nr. 12 ist alles Defizit „öffentlich“, das „zum Staat, d.h. zum Zentralstaat, zu regionalen oder lokalen Gebietskörperschaften oder Sozialversicherungseinrichtungen gehört, mit Ausnahme von kommerziellen Transaktionen im Sinne des Europäischen Systems volkswirtschaftlicher Gesamtrechnung.

30 Schulze Steinen, M., a.a.O., 224. 
den Grenzen des Art. 126 Abs. 1 AEUV liegende Gesamtschuldenstände möglicherweise refinanzierbar sein können.

\section{c.) Die Reform von 2005}

$\mathrm{Zu}$ diesen generellen Mängeln trat im Jahre 2005 eine Reform, deren erklärtes Ziel es war, „haushaltspolitische Flexibilität“" zu gewinnen. ${ }^{31}$ Die Begrenzung dieser Flexibilität sah der Rat aber nicht in der schon 2005 gewaltigen Schuldenlast, sondern in den Restriktionen durch das Europäische Primär- und Sekundärrecht.

Dementsprechend höhlt die VO 1056-2005 den Defizitbegriff aus, indem gewisse Ausgaben bei der Defizitberechnung nicht mehr $\mathrm{zu}$ berücksichtigen sind. Darüber hinaus wurden die Ermessensspielräume von Kommission und Rat durch die Reform ausgedehnt ${ }^{32}$ und die Ausnahmetatbestände des Art. 126 Abs. 2 AEUV erweitert. ${ }^{33}$ Prozedural führte die Reform dazu, dass nun von der Mitteilung der Haushaltsdaten der Mitgliedstaaten bis zum Beschluss über mögliche Sanktionen sechzehn statt der ursprünglich intendierten zehn Monate vergehen. ${ }^{34}$ Alle bisherigen Verfahren endeten sanktionslos und waren stets von längerer Dauer. ${ }^{35}$

\section{Rechtliche Verbindlichkeit der Vorgaben}

Art. 126 Abs.1 AEUV konstituiert mit der Aussage „Die Mitgliedstaaten vermeiden übermäßige öffentliche Defizite“ eine echte Rechtspflicht. ${ }^{36}$

Entgegen dem Wortlaut ist hierfür aber nicht allein das Finanzierungsdefizit entscheidend. Das Ziel ist stattdessen eine auf Dauer tragbare Gesamtfinanzlage der öffentlichen Hand. ${ }^{37}$ Das ergibt sich aus der Bezeichnung ,öffentliches Defizit“" in Art.126 Abs.6 AEUV. Zu seiner Bestimmung soll auf die Kriterien nach

31 Mit Verweis auf die Rolle Deutschlands und Frankreichs im Reformprozess: Hentschelmann, K., a.a.O., 1687.

32 Häde U., Art.126 AEUV, Rn.112, in: Callies/Ruffert: EUV/AEUV Kommentar, München 2011.

33 Art. 1 Nr. 1 Abs. 3 VO 1056/05.

34 15. Erwägungsgrund der VO 1467/97 in der ursprünglichen Fassung.

35 Häde,U. aaO., Art. 126 AEUV Rn.109.

36 Allgemeine Ansicht, u.a. Streinz/Ohler/Herrmann: Totgesagte leben länger - oder doch nicht? Der Stabilitäts- und Wachstumspakt nach dem Beschluss des Rates vom 25. November 2004 über das Ruhen der Defizitverfahren gegen Frankreich und Deutschland, in: Neue Juristische Wochenschrift, 2004, 1553-1559, 1554.

37 Palm, U.: Preisstabilität in der Europäischen Wirtschafts- und Währungsunion, Baden-Baden, 2000, $150 \mathrm{ff}$. 
Abs.2 zurückgegriffen werden. Dort wird neben dem Defizit aber auch der Gesamtschuldenstand berücksichtigt und es erfolgt eine „Prüfung der Gesamtlage“, also eine Gesamtbeurteilung der Haushaltsdisziplin. Weil das Sanktionsverfahren der Abs. 8-12 nur an diese Feststellung anknüpfen kann, ist Abs. 1 weit zu verstehen, umfasst also auch den Gesamtschuldenstand.

\section{Rechtliche Durchsetzbarkeit der Vorgaben}

Da Art. 126 Abs.1 AEUV eine echte Rechtspflicht konstituiert, ergibt sich die Frage, wem in welchem Verfahren die Feststellung ihrer Einhaltung obliegt.

\section{Vertragsverletzungsverfahren}

Verpflichtete des Art. 126 AEUV sind die Mitgliedstaaten. Jeder Verstoß ihrerseits stellt demnach eine Vertragsverletzung dar, die jeder andere Mitgliedstaat oder die Kommission im Vertragsverletzungsverfahren (Art. 258, 259 AEUV) geltend machen könnte. Art. 126 Abs. 10 AEUV schließt dieses Recht jedoch aus. So bleibt nur das spezielle Defizitverfahren des Art. 126 Abs. 3-9 AEUV, um einen Mitgliedstaat zum Tätigwerden zu veranlassen.

\section{Defizitverfahren}

Das Defizitverfahren unterteilt sich in das Überwachungsverfahren nach Abs.3-5 und das Feststellungs- und Sanktionsverfahren nach Abs. 6-9.

\section{a.) Aufgabenverteilung}

Der Kommission als Hüterin der Verträge kommen dabei die Initiativ- und Überwachungsrechte zu. Rechtsverbindliche Entscheidungen trifft aber allein der Rat. $^{38}$ Dessen Mitglieder sind die Fachminister der Mitgliedstaaten, Art. 16 II EUV. Über die Vertragsverletzung entscheiden also nicht die Richter des EuGH oder des EuG, sondern die Finanzminister der Mitgliedstaaten. Sie richten so über die Verfehlungen ihres Kollegen. Dieser selbst ist als Ratsmitglied, das den betroffenen Mitgliedstaat vertritt, bei den Beschlussfassungen nicht stimmberechtigt, Art. 126 Abs. 13 UAbs. 2 AEUV.

38 EuGH, Urteil vom 13.7.2004, C 27/04, Rn.76: „liegt die Verantwortung für die Wahrung der Haushaltsdisziplin im Wesentlichen beim Rat.“. 


\section{b.) Beurteilungsspielräume und Justiziabilität der Entscheidungen des Rates}

Auch der Rat ist aber den Bestimmungen des AEUV unterworfen. Fraglich ist deshalb, inwiefern er den Einschätzungen der Kommission Folge leisten muss oder seine eigenen Bewertungen der Sachlage als maßgeblich erachten darf. Grundsätzlich ist schon das letztlich maßgebliche Kriterium der „wirtschaftliche Gesamtlage" denkbar weit und unbestimmt gefasst, sodass dem Rat ein Beurteilungsspielraum zusteht. ${ }^{39}$ Selbst die konkret fassbaren Referenzkriterien werden aber durch die mit unbestimmten Rechtsbegriffen gespickten Ausnahmeregelungen des Art. 126 II AEUV aufgeweicht (u.a.: „,kontinuierlicher Rückgang“, ,,ausnahmsweise und vorübergehend überschritten"), bei deren Vorliegen abermals Ermessensspielräume bestehen. Die Reform von 2005 vergrößerte diese Beurteilungsspielräume weiter.

\section{c.) Reduzierte Spielräume?}

Aus der Bindung des Rates an Art. 126 Abs.1 AEUV erwächst die Frage, ob seine Beurteilungsspielräume unbeschränkt bestehen oder ob er unter bestimmten Bedingungen gezwungen sein kann, zu beschließen, dass ein übermäßiges Defizit besteht und die entsprechenden Sanktionen zu verhängen sind. Sollte eine solche Ermessensreduzierung vorliegen und der Rat trotzdem nicht das Bestehen eines Defizits beschließen, wäre prozessual eine Untätigkeitsklage statthaft.

Im oben beschriebenen Urteil von 2004 hat der EuGH die Haushaltsdisziplin als Voraussetzung für die Sicherung der Preisstabilität anerkannt. Folglich seien Art. 126 AEUV und VO 1467/97 so auszulegen, dass ihre volle praktische Wirksamkeit gesichert und damit das Ziel der Preisstabilität erreicht werde. ${ }^{40} \mathrm{Im}$ selben Urteil weist der EuGH die Kommission auf die Möglichkeit einer solchen Untätigkeitsklage hin und betont, mit dem Urteil zur vorliegenden Nichtigkeitsklage nicht über derartige Beschlusspflicht entschieden zu haben. ${ }^{41}$

Die Verpflichtung des Rates auf das Ziel der Preisstabilität könnte demnach dazu führen, dass dieser nicht in Gänze frei entscheiden kann, sondern bei einer eklatant untragbaren Gesamtsituation der öffentlichen Finanzen gezwungen ist, einen Verstoß gegen Art. 126 Abs. 1 AEUV festzustellen und entsprechende Sanktionen zu beschließen. ${ }^{42}$

39 Ebd., Rn. 80.

40 Ebd., Rn. 74.

41 Ebd., Rn. 90.

42 Badilla, R.: Stabilitäts- und Wachstumspakt, a.a.O., 547; eine Ermessenseinschränkung befürworten Streinz/Ohler/Herrmann, a.a.O. 1556, sowie Häde, U., a.a.O., Art. 126 Rn.27. 
So begründet Art. 126 Abs. 1 AEUV eine echte Rechtspflicht der Mitgliedstaaten, für zukunftsfähige öffentliche Haushalte zu sorgen. Die dafür im Protokoll Nr. 12 genannten Werte von Gesamtschuldenstand und Neuverschuldung sind nicht alleine maßgeblich, stellen aber wichtige Referenzkriterien dar. Beide Kriterien sind ökonomisch nicht zwingend und im hohen Maße interdependent. Probleme im Vergleich der einzelnen Staatshaushalte schwächen ihre Autorität weiter.

Zur Durchsetzung des Zieles aus Art. 126 Abs. 1 AEUV kommt einzig ein Defizitverfahren in Betracht. Initiativorgan dieses Verfahrens ist die Kommission, das Beschlussorgan der Rat. So entscheiden Finanzpolitiker statt Richtern über die Einhaltung von Rechtsnormen. Der Rat ist nur in geringem Umfang an die Vorgaben der Kommission gebunden und verfügt über weite Beurteilungs- und Ermessensspielräume. Diese Spielräume werden durch die Bindung des Rates an die Preisstabilität nur schwach begrenzt.

\section{Aktuelle Entwicklungen}

Die Finanzkrise hat das politische Bewusstsein für die Gefahr überschuldeter öffentlicher Haushalte gestärkt und so Überlegungen angeregt, die Defizite wirksamer zu kontrollieren.

\section{Vorschläge der Van Rompuy-Gruppe}

Nach den Vorschlägen der Van Rompuy-Gruppe sollen die ökonomischen Ungleichgewichte in den Mitgliedstaaten stärker überwacht werden. Dabei wird dem Kriterium des Gesamtschuldenstandes eine größere Aufmerksamkeit zuteil. ${ }^{43}$ Zudem soll das Sanktionsinstrumentarium bei Verstößen erweitert und verschärft werden. ${ }^{44}$ Des Weiteren ist angedacht, Sanktionen künftig stärker automatisiert ablaufen zu lassen, indem der Rat den Vorschläge der Kommission mit qualifizierter Mehrheit widersprechen muss, um ihren Vollzug zu verhindern (sog. Widerspruchslösung). ${ }^{45}$

43 Dokument 15302/10 des Rates der Europäischen Union vom 21.10.2010, Rn.6 ff.

44 Ebd., Rn.16ff.

45 Ebd., Rn.24ff. 
Soweit ersichtlich, beziehen sich diese Maßnahmen aber weiterhin nur auf das Sanktionsverfahren, also die Phase nach Feststellung eines Verstoßes. ${ }^{46}$ Die Probleme bei der Feststellung selbst bleiben damit unbearbeitet.

\section{Vorschläge der Kommission}

Die Vorschläge der Kommission ähneln jenen der Van Rompuy-Gruppe. So plädiert auch sie für eine stärkere Berücksichtigung des Schuldenstandes ${ }^{47}$ und eine stärkere Automatisierung der Sanktionen durch die bereits vorgestellte Widerspruchslösung. Außerdem spricht sich die Kommission für eine neue Richtlinie zur Vermeidung makroökonomischer Ungleichgewichte inklusive eines entsprechenden Zwangsmechanismus aus, ohne diesen näher zu präzisieren. ${ }^{48}$

\section{3. Überblick über die Gipfelbeschlüsse des Jahres 2011}

Gestützt auf diese Vorarbeiten durchzog eine Reihe sog. „Euro-Gipfel“ das europapolitische Jahr 2011. Ihr Haupttagesordnungspunkt war die unmittelbare Krisenbewältigung, wobei Maßnahmen zur Liquiditätssicherung insbesondere Griechenlands im Vordergrund standen. So wurde etwa der Europäische Stabilitätsmechanismus (ESM) auf dem Brüsseler Gipfel vom 24.-25.3. verstetigt, was bei den Mechanismen zur Sicherung der Haushaltsstabilität der Mitgliedstaaten insofern zu einer minimalen Änderung führte, als die Höhe der Bußgelder für die Nichteinhaltung der Kriterien fortan dem ESM überlassen werden. ${ }^{49}$

Zudem ergingen einige wegweisende Beschlüsse zur zukünftigen Sicherung der gemeinsamen Währung. ${ }^{50}$ Darunter sind vor allem zu nennen:

- die geänderte Verordnung (EG) Nr. 1466/97 „über den Ausbau der haushaltspolitischen Überwachung und der Überwachung und Koordinierung der Wirtschaftspolitiken";

- die Verordnung (EG) des Parlaments und des Rates „über die wirksame Durchsetzung der haushaltspolitischen Überwachung im Euroraum“;

46 Ebd., Rn.21ff.

47 Pressemitteilung der Kommission, 10/1199, Punkt 2.

48 Ebd., Punkte 5 und 6.

49 Schlussfolgerungen des Rates vom 20.4.2011, EUCO 10/1/11 REV 1, 22.

50 Ausführlicher Überblick und Kritik bei: Weber: Die Reform der Wirtschafts- und Währungsunion in der Finanzkrise, in: Europäische Zeitschrift für Wirtschaftsrecht, 2011, 935-940; Horn, N.: Die Reform der Europäischen Währungsunion und die Zukunft des Euro, in: Neue Juristische Wochenschrift, 20 (2011), 1398-1404. 
- $\quad$ eine Richtlinie des Rates über die Anforderungen an die haushaltspolitischen Rahmen der Mitgliedstaaten;

- die Verordnung (EG) des Parlaments und des Rates vom 28.10.2011 „über die Vermeidung und Korrektur makroökonomischer Ungleichgewichte"; sowie

- die Verordnung (EG) vom 28.10.2011, „über Durchsetzungsmaßnahmen zur Korrektur übermäßiger makroökonomischer Ungleichgewichte.

Die europäische Antwort besteht also in verstärkter gemeinschaftlicher Absprache und gemeinsamer Aufsicht sowie schärferen Zwangsmaßnahmen gegenüber einzelnen Mitgliedstaaten. Aus der dargelegten Abhängigkeit des Schuldenstandes vom BIP folgt, dass neben Sparanstrengungen auch Wachstum ein Weg aus der Krise wäre. Entsprechende Überlegungen waren in den letzten Monaten selten, scheinen nun aber wieder salonfähiger zu werden. ${ }^{51}$

Obwohl eine Reihe von Vorschlägen durchaus erfolgversprechend erscheint, bleiben bei allem Reformeifer doch gravierende Probleme bestehen. Striktere oder gar zwangsweise durchsetzbare Haushaltsregelungen auf europäischer Ebene gehen zu Lasten nationaler Souveränität und begegnen damit Bedenken des nationalen Verfassungsrechts. ${ }^{52}$ Im Europarecht gilt das Prinzip der begrenzten Einzelermächtigung - sofern die Verträge eine solche nicht enthalten, bedarf es der aus der historischen Erfahrung sehr schwierigen und zeitaufwendigen Änderung des europäischen Primärrechts. ${ }^{53}$

Von diesen Problemen bei der Umsetzung der neuen Regelungen abgesehen, muss die nun vorgeschlagene nationalverfassungsrechtliche „Schuldenbremse“ ihre positive Wirkung in Deutschland erst noch beweisen. Im Ergebnis bleibt zu hoffen, dass es mit der europaweiten Einrichtung nationaler Stabilitätsvorschriften gelingt, der grassierenden Staatsverschuldung in den Mitgliedstaaten Einhalt zu gebieten.

51 Vgl. etwa Punkt 4 der Pressemitteilung 125672/2011 des Rates.

$52 \mathrm{Zu}$ entsprechenden Andeutungen deutscher Verfassungsrichter: Herdegen, Handelsblatt vom 14.1.2012, $14 \mathrm{f}$.

53 Beispiele für solche Probleme im Rahmen der benannten Rechtsakte finden sich bei Weber, a.a.O., 2011, 936f. 